\title{
Patterning artificial lipid bilayer on nanostructured surfaces
}

This article was published in the following Dove Press journal:

International Journal of Nanomedicine

\author{
Ranjita Ghosh Moulick' \\ Gregor Panaitov ${ }^{2}$ \\ Sung-Eun Choi ${ }^{2}$ \\ Dirk Mayer ${ }^{2}$ \\ Andreas Offenhäusser ${ }^{2}$ \\ 'School of Physical Science, \\ Jawaharlal Nehru University, \\ New Delhi, India; ${ }^{2}$ Bioelectronics, \\ ICS8/PGI8, Forschungszentrum \\ Juelich, Juelich, Germany
}

\begin{abstract}
Artificial lipid bilayer on solid substrate plays an important role as an interface between nanotechnology and biology. In this study, grid structures were patterned on Au$\mathrm{Nb}$-glass substrate and artificial bilayer was prepared on these structures. The fluidity was checked using fluorescence recovery after photobleaching (FRAP), and neuronal adhesion was monitored on such structure using EphrinA5-tethered lipid bilayer. EphrinA5 is a ligand that binds to the Eph receptors of rat cortical neurons and influences cellular adhesion. Our result elucidated that influence of these nanopatterned protein-tethered lipid bilayer on cellular guidance and signaling can address many underlying mechanisms of cellular functioning and help us to understand and differentiate the signaling procedure in cancer and neurodegenerative diseases.
\end{abstract}

Keywords: Au-Nb, FRAP, EphrinA5, lipid bilayer, neurodegenerative diseases

\section{Introduction}

Artificial lipid bilayer on solid substrate plays an important role as an interface between nanotechnology and biology. This biomimetic membrane serves as an excellent model system to study cellular signaling and guidance. For signaling, cells confine the lipids and membrane proteins into specific domain, known as membrane domain. ${ }^{1}$ The lateral diffusion of the membrane, due to its fluidic nature, is responsible for such spatial organization and compartmentalization of the signaling molecules. Artificially prepared lipid bilayer also retains this property. ${ }^{2}$ By using barriers or constraints on the surfaces, this fluidity can be restricted, and both lipid and lipid-tethered proteins can be compartmentalized within these domains. ${ }^{3}$ In this study, grid structures were patterned on $\mathrm{Au}-\mathrm{Nb}$-glass substrate and artificial bilayer was prepared on these structures. The fluidity was checked using fluorescence recovery after photobleaching (FRAP), and neuronal adhesion was monitored on such structure using EphrinA5-tethered lipid bilayer. ${ }^{4}$ EphrinA5 is a ligand that binds to the Eph receptors of rat cortical neurons and influences cellular adhesion. ${ }^{5}$

\section{Materials and methods}

Fabrication of structures and characterization using scanning electron microscopy (SEM): 1-2 nm of $\mathrm{Nb}$ was deposited on glass surface prior to the coating of the photoresist, poly(methyl methacrylate) (PMMA). By using e-beam writer, the desired structures were transposed on the PMMA surface. PMMA was developed, and successively Au was deposited. The undesired Au was removed using the liftoff technique. Finally, the $\mathrm{Nb}$ was removed by reactive ion etching. SEM (FEI, Hillsboro, OR, USA) was performed to characterize the structures.
Correspondence: Ranjita Ghosh Moulick School of Physical Science, Jawaharlal Nehru University, New Delhi I 10067 India

Email ranjita.ghoshmoulick@gmail.com 


\section{Bilayer preparation and FRAP}

1-Palmitoyl-2-oleoyl-sn-glycero-3-phosphocholine (POPC) was dried under vacuum on the wall of a glass vessel, $0.1 \mathrm{M}$ phosphate-buffered saline (PBS) or EphrinA5 was dissolved in detergent, and 0.1 M PBS was added to this dried lipid, vortexed, and sonicated to prepare liposomes or proteoliposomes, finally extruded through $100 \mathrm{~nm}$ membrane by Avanti mini extruder (Avanti Polar Lipids, Inc) to get small unilamellar vesicles (SUVs). These SUVs were fused on the cleaned and plasma activated patterned surface to get lipid bilayer. For FRAP 5\% Oregon Green 1,2-dihexadecanoylsn-glycero-3-phosphoethanolamine (Oregon Green 488 DHPE) doped POPC-SUVs were deposited on the patterned surface. Bleaching was done by using a 473-nm laser from Rapp OptoElectronics coupled into a Zeiss Axio Scope, and a series of images were made to track the recovery of fluorescence.

\section{Neuronal adhesion on EphrinA5-tethered lipid bilayer}

About $40,000 / \mathrm{mL}$ neurons were cultured on EphrinA5 incorporated lipid bilayer. Bright field images were captured just after 4 hours of cell adhesion with and without EphrinA5-tethered lipid bilayer.

\section{Results and discussion}

Figure 1 shows a schematic representation of the Au grid pattern on the glass surface and SEM images of the pattern. Grid structures made of $\mathrm{Au}$ (wall of $10 \mathrm{~nm}$ in height and $0.2 \mu \mathrm{m}$ in width) in 5 different ranges $(2.5,2,1.5,1$, and $0.5 \mu \mathrm{m})$ were fabricated on a glass substrate with a spacing of $10 \mu \mathrm{m}$ between them. All these grids were fabricated in arrays. The SEM images show different regions of the patterns after the fabrication step. The white lines in the images show the Au walls of the structures. Five percent Oregon Green doped POPC-SUVs were deposited on the patterned surface for bilayer preparation and fluorescence imaging for FRAP experiments. Bleaching was done using a laser and recovery was monitored by capturing the images of the same area at different time intervals. FRAP was done for each region of the grid structures, and it was found that the lipids are mobile within the grids (Figure 2). This could be accounted for two reasons. First, there could be nano gaps within the grids through which lipids can move from
A

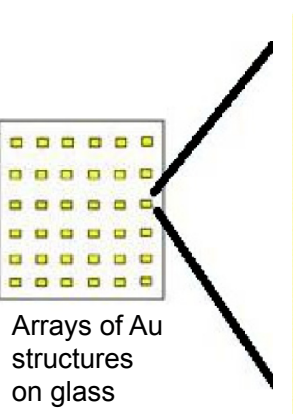
each structures

C

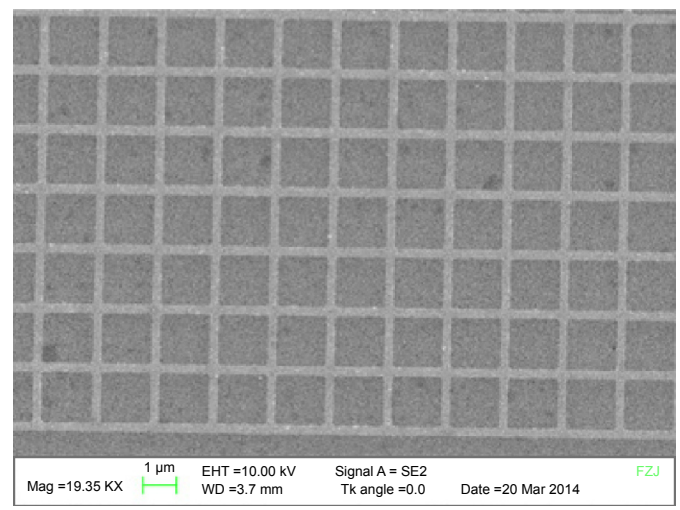

$0.5 \mu \mathrm{m}$

$1 \mu \mathrm{m}$

$1.5 \mu \mathrm{m}$

$2 \mu \mathrm{m}$

$2.5 \mu \mathrm{m}$

\section{With a gap of $10 \mu \mathrm{m}$ between}

D

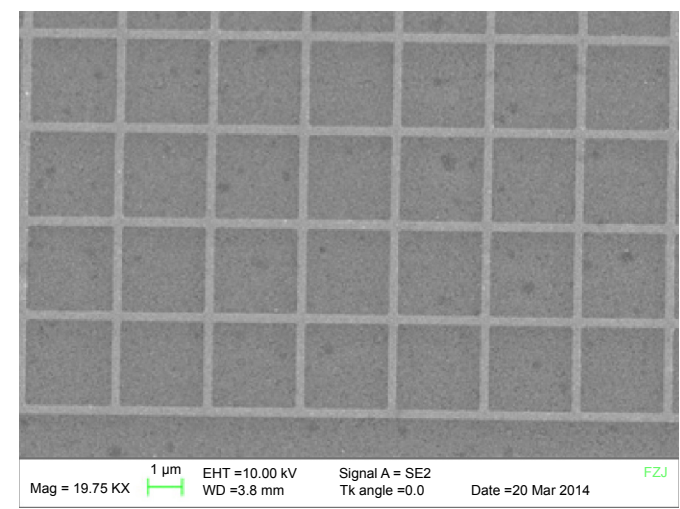

B

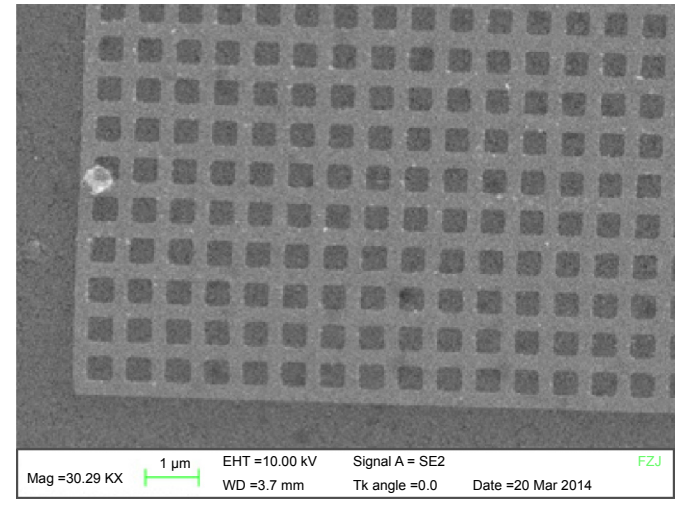

D

Figure I Schematics of the grid pattern (A) and the scanning electron microscope images of the patterns with $0.5,1.5$, and $2.5 \mu \mathrm{m}$ grid $(\mathbf{B}-\mathbf{D}$, respectively). 

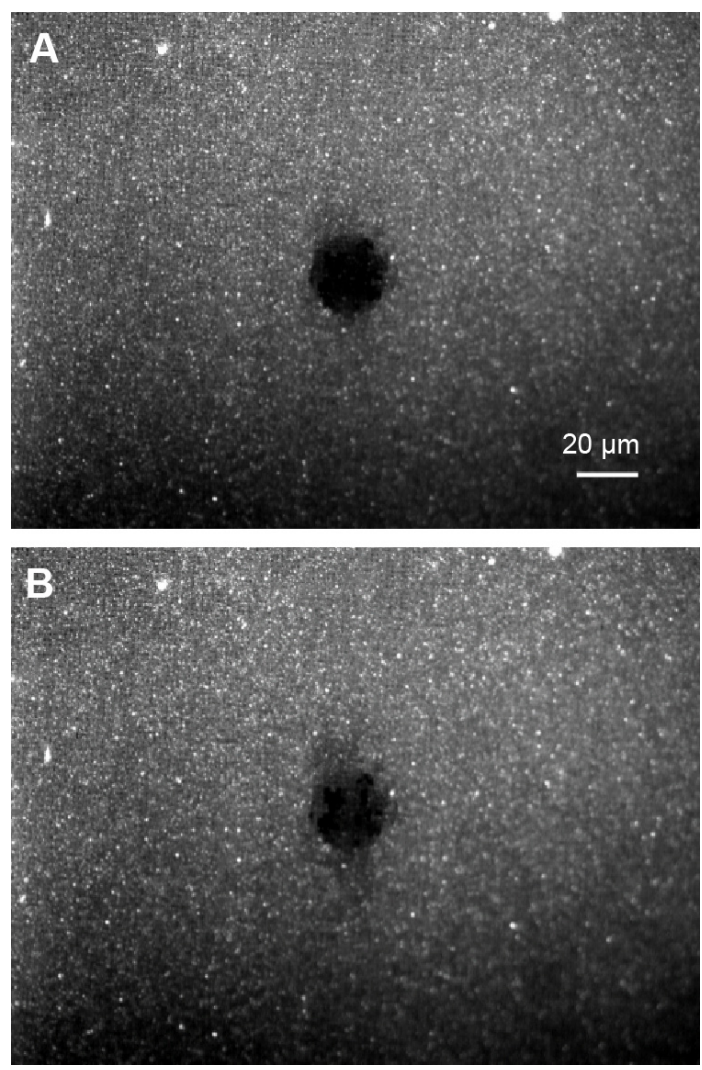

Figure 2 Fluorescence recovery after photobleaching of Oregon Green I,2dihexadecanoyl-sn-glycero-3-phosphoethanolamine doped lipid bilayer in the length/ breadth of $1.5 \mu \mathrm{m}$ grid.

Notes: Images captured just after bleaching (A) and $42 \mathrm{I}$ seconds after bleaching (B).

one grid to another because there is always a possibility that during liftoff or cleaning process, chunks of Au are removed, which affect the homogeneity of the structure and create the nano gaps in the grid wall. However, 15 -nm height could be too low for the lipids, and due to their lateral mobility, they could move from one compartment to another.

To check the distribution and diffusion in this guided geometry, a cell signaling protein EphrinA5 was incorporated into the model membrane. Rat cortical neurons were cultured on these EphrinA5-tethered lipid bilayer prepared on the patterned surface. Neuronal adhesion was monitored just after 4 hours of neuronal plating. Neuronal aggregation is observed on such patterned EphrinA5-tethered lipid bilayer (Figure 3).

\section{Conclusion}

In this report, a prepatterned glass substrate with Au structures in the range of nanometers is used for artificial lipid bilayer preparation. Repeated experiments of FRAP showed that the lipids are mobile and they can move within the structures. EphrinA5-tethered lipid bilayer can promote neuronal adhesion on
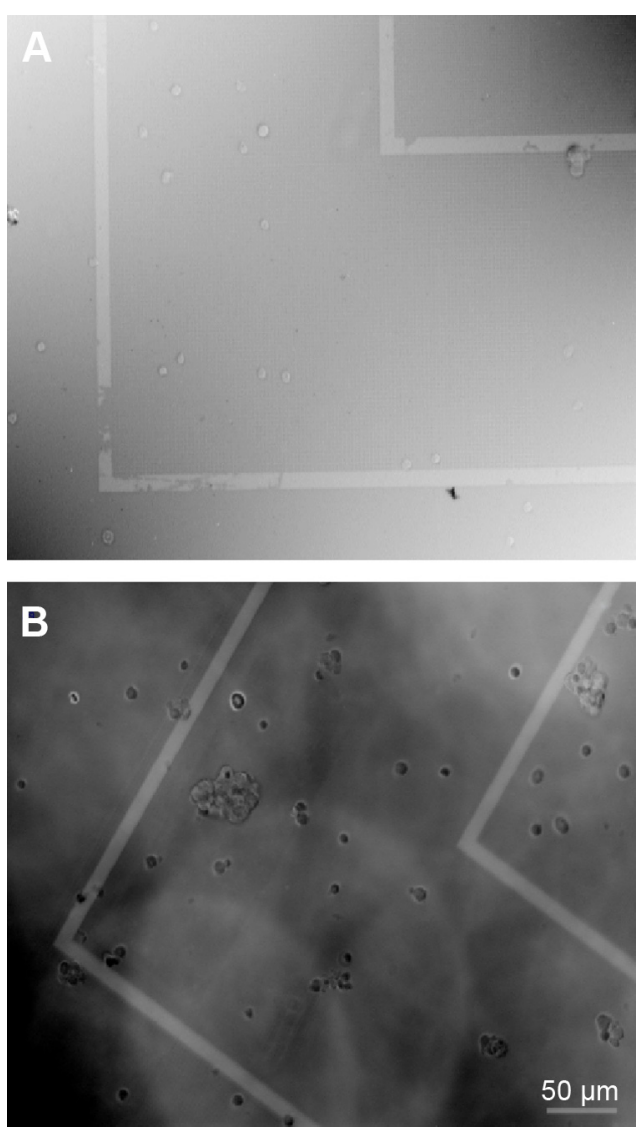

Figure 3 Neuronal culture on control substrate (A) and EphrinA5-tethered lipid bilayer (B).

these structures. The influence of these nanopatterned proteintethered lipid bilayer on cellular guidance and signaling can address many underlying mechanisms of cellular functioning and help us to understand and differentiate the signaling procedure in cancer and neurodegenerative diseases.

\section{Disclosure}

The authors report no conflicts of interest in this work.

\section{References}

1. Mukherjee S, Maxfield FR. Membrane domains. Annu Rev Cell Dev Biol. 2004;20:839-866.

2. Cremer PS, Boxer SG. Formation and spreading of lipid bilayers on planar glass supports. J Phys Chem B. 1999;103:2554-2559.

3. Nair PM, Salaita K, Petit RS, Groves JT. Using patterned supported lipid bilayer membranes to investigate the role of receptor organization in intercellular signaling. Nat Protoc. 2011;6(4):523-539.

4. Ghosh Moulick R, Afanasenkau D, Choi SE, et al. Reconstitution of fusion proteins in supported lipid bilayers for the study of cell surface receptor-ligand interactions in cell-cell contact. Langmuir. 2016; 32(14):3462-3469

5. Klein R. Bidirectional modulation of synaptic functions by Eph/ephrin signaling. Nat Neurosci. 2008;12(1):15-20. 


\section{Publish your work in this journal}

The International Journal of Nanomedicine is an international, peerreviewed journal focusing on the application of nanotechnology in diagnostics, therapeutics, and drug delivery systems throughout the biomedical field. This journal is indexed on PubMed Central, MedLine, CAS, SciSearch $\AA$, Current Contents $\AA /$ Clinical Medicine,

Journal Citation Reports/Science Edition, EMBase, Scopus and the Elsevier Bibliographic databases. The manuscript management system is completely online and includes a very quick and fair peer-review system, which is all easy to use. Visit http://www.dovepress.com/ testimonials.php to read real quotes from published authors.

Submit your manuscript here: http://www.dovepress.com/international-journal-of-nanomedicine-journal 